\title{
Tunable Terahertz Meta-Surface with Graphene Cut-Wires
}

\author{
Yuancheng Fan, ${ }^{* \dagger}$ Nian-Hai Shen, ${ }^{*} \neq$ Thomas Koschny, ${ }^{\ddagger}$ and Costas M. Soukoulis ${ }^{\ddagger} \S$ \\ ${ }^{\dagger}$ Key Laboratory of Space Applied Physics and Chemistry, Ministry of Education and Department of Applied Physics, School of \\ Science, Northwestern Polytechnical University, Xi'an 710072, China \\ ${ }^{\ddagger}$ Ames Laboratory and Department of Physics and Astronomy, Iowa State University, Ames, Iowa 50011, United States \\ ${ }^{\S}$ Institute of Electronic Structure and Laser, FORTH, 71110 Heraklion, Crete, Greece
}

ABSTRACT: We propose a tunable meta-surface in the terahertz regime by patterning a graphene sheet in cut-wire array. The enhancement of terahertz absorption of such a graphene meta-surface was studied detailedly via the optimization to the geometry of the structure. Considering the data of graphene in both the experimental and theoretical perspectives, we investigated the performance of the absorbing graphene meta-surface by extracting its effective surface conductivities through a sheet retrieval method. We also specifically considered two sets of well-known experimental graphene data and comparatively studied the properties of graphene meta-surface by changing the graphene parameters inbetween. It shows that there has been significant improvement in preparing high-quality graphene samples, which makes it possible to strengthen optical properties of graphene microstructures, and therefore benefits various practical applications.

KEYWORDS: graphene, terahertz, metamaterials, plasmonics, absorption enhancement, effective surface conductivity
M etamaterials refer to artificial structures with properties beyond natural materials. ${ }^{1,2}$ These artificial materials usually consist of subwavelength-sized resonant building blocks as their "elementary particles" (known as meta-atoms), which yield exotic electric and magnetic responses while interacting with electromagnetic (EM) fields. A kind of single-layer metamaterial, namely, meta-surface, ${ }^{3,4}$ has drawn enormous attention in recent years for various possibilities to manipulate light with ultrathin structured surfaces. Tailored planar "metaatoms" of the meta-surfaces can be exploited to realize abrupt discontinuities for manipulating light beams peculiarly, for example, for generalized Snell's law, ${ }^{5}$ broadband light bending, ${ }^{6}$ optical morphology conversion, ${ }^{7}$ photonic spin Hall effect, and spin-controlled photonics. ${ }^{8,9}$ Incorporating tunability and switching mechanism into the planar systems is one important issue to address to take further advantage of metasurfaces. $^{3,10,11}$

A monolayer of carbon atoms in a hexagonal lattice with high-performance electric, thermal, and mechanic properties, graphene, has recently attracted considerable attention for both its fundamental physics and enormous applications. Such a realistic two-dimensional (2D) electron gas or two-dimensional material emerges in photonics and optoelectronics. ${ }^{12-15}$ As a new optoelectronic material, graphene exhibits much higher confinement of surface plasmon polaritons and supports relatively longer propagation. ${ }^{16-22}$ Linear dispersion of the 2D Dirac Fermions provides ultrawideband tunability under various implementations, such as the application of electrostatic field, magnetic field, or chemical doping. ${ }^{21-26}$ However, since graphene is a monolayer atomic sheet with relatively low carrier concentrations, its interaction with light is not very strong; for example, graphene is almost transparent to optical waves. ${ }^{27}$ Artificially constructed metamaterial structures, as a platform for enhancing light-matter interactions, ${ }^{28,29}$ have been employed to strengthen graphene's ability in the manipulation of light signals. A recent study reported that optical absorption enhancement can be achieved with periodic nanodisks of doped-graphene on a dielectric substrate or film coating on metal, and the excitation of the electric mode of the nanodisks together with multiple reflections from the assistance of total internal reflection and metal reflection can result in a complete optical absorption. ${ }^{30}$ Various configurations with graphene, such as micro/nanoribbons, mantles, nanocrosses, ring resonators, coherent modulations, and superlattices, have also been investigated in the manipulation of the terahertz $(\mathrm{THz}) /$ infrared waves, for the enhancement of absorption, polarization conversion, cloaking, and so on. ${ }^{31-40}$ Different from these previous studies, here we stick with the most fundamental electric dipolar mode, which is found to provide stronger lightgraphene interaction at $\mathrm{THz}$ frequencies than the magnetic and higher-order electric modes, and we mainly focus on strengthening $\mathrm{THz}$ response of patterned graphene at the electric dipolar excitation based on the investigations to the relevant graphene parameters. In this work, we propose a tunable meta-surface by exploring a monolayer graphene patterned in an array of cut-wires, the simplest structure supporting the electric dipolar mode that has been widely adopted in exploring fundamental physics, as well as novel

Received: October 3, 2014

Published: December 22, 2014 
functionalities, such as plasmon-induced transparency, ${ }^{41-43}$ polarization manipulations, ${ }^{44}$ and optical antennas. ${ }^{45} \mathrm{We}$ demonstrate a $50 \%$ maximum absorption arising from the electric dipolar mode, which is confirmed by the extraction of effective surface conductivities of cut-wire array of theoretical and experimental graphene. Systematic investigations to the graphene meta-surface by changing the collision frequency and Drude weight (see below) of graphene with the values between two sets of well-known experimental data, that is, named as $\mathrm{Li}$ et al. data ${ }^{23}$ and Yan et al. data ${ }^{13}$ further on, respectively, show that optical response of the graphene cut-wire-based metasurface can be tuned substantially in the $\mathrm{THz}$ regime.

Figure 1 shows the schematic of the proposed tunable graphene meta-surface. The meta-atoms, that is, graphene cut-

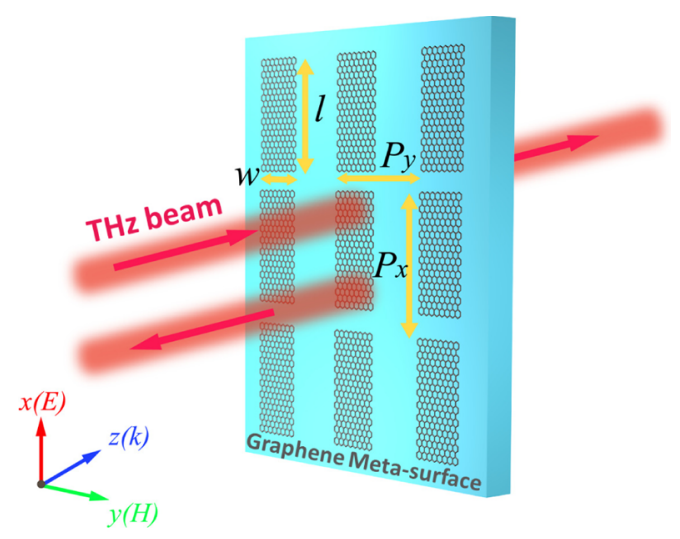

Figure 1. Schematic of the single-layer graphene cut-wire structure together with the corresponding excitation configuration: $x$-polarized (electric field $E$ is along the $x$-axis) incident light is propagating along the $z$-axis. Geometric parameters are denoted by black letters.

wires, are periodically arranged in the $x-y$ plane with lattice constants $P_{x}=6.0 \mu \mathrm{m}$ and $P_{y}=3.0 \mu \mathrm{m}$. The length and width of the cut-wire meta-atoms are $l$ and $w$, respectively, which we will find both play important roles in determining the EM properties of graphene meta-surface. In the setup, the $\mathrm{THz}$ beam, with electric field polarized along $x$-direction, normally illuminates graphene meta-surface along the $z$-axis (see Figure 1).

In our numerical calculations, the graphene layer is reasonably treated as a sheet material modeled with complex surface conductivity $\left(\sigma_{\mathrm{g}}\right)$ since a one-atom-thick graphene sheet is sufficiently thin compared with the concerned wavelength. In the theoretical perspective based on random-phase-approximation (RPA), ${ }^{46-48}$ the complex conductivity of graphene can be described by the Drude model as $\sigma_{\mathrm{g}}=i e^{2} E_{\mathrm{F}} / \pi \hbar^{2}\left(\omega+i \tau^{-1}\right)$, especially in the heavily doped region and low frequencies (far below Fermi energy), where $E_{\mathrm{F}}$ represents the Fermi energy, $\tau$ $=\mu E_{\mathrm{F}} / e v_{\mathrm{F}}^{2}$ is the relaxation rate with the mobility $\mu=10^{4} \mathrm{~cm}^{2}$ $\mathrm{V}^{-1} \mathrm{~s}^{-1}$ and Fermi velocity $v_{\mathrm{F}} \approx 10^{6} \mathrm{~m} / \mathrm{s}$. For simplicity, we rewrite the expression of surface conductivity in the form as $\sigma_{\mathrm{g}}$ $=\alpha /(\gamma-i \omega)$, in which $\alpha$ is the so-called Drude weight and $\gamma$ is the collision frequency. ${ }^{49}$ With respect to the simulations, we adopted the well-known commercial electromagnetic solver, that is, CST MICROWAVE STUDIO.

We first took $E_{\mathrm{F}}=0.5 \mathrm{eV}$, as that in ref 40 , for the graphene. Figure 2a presents the spectra of transmission $(T)$, reflection $(R)$, and absorption $(A)$ spectra for the case of graphene cutwire meta-surface with width of wire $w=0.7 \mu \mathrm{m}$ and length $l=$ $5 \mu \mathrm{m}$. According to it, an obvious resonance exists around 3.30
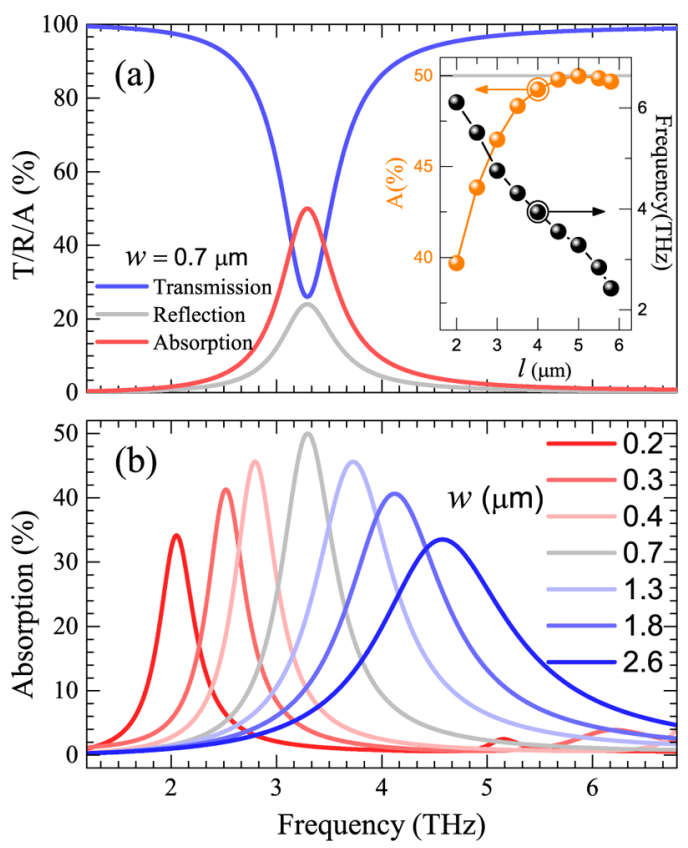

Figure 2. (a) Transmission, reflection, and the absorption of a graphene cut-wire array $(w=0.7 \mu \mathrm{m})$. (b) The absorption spectra for graphene cut-wire arrays with different cut-wire widths: the resonant spectra show a blue shift (from red to blue) regarding the increasing of the widths. Optical conductivity of the graphene is set with the Drude weight $\alpha=58.86 \mathrm{GHz} / \Omega$ and the collision frequency $\gamma=2 \mathrm{THz}$. The inset in (a) shows the length dependence of the peak absorption and resonance frequency for graphene cut-wire array with $w=0.7 \mu \mathrm{m}$.

THz. The resonance was confirmed to be electric dipolar mode through the field and current distributions (results not shown here), similar to the low-frequency resonance of split-ringresonators, as shown in ref 38 , and the excitation of such a resonance leads to the enhancement of absorption in the graphene sheet. Figure $2 b$, that is, the absorption spectra of graphene meta-surface with different $w$ shows the influence of line width of cut wire to the $\mathrm{THz}$ response of graphene metasurface: It is found that the resonance frequency of the electric dipolar mode shows a monotonous blue-shift with the increase of $w$, while there exists an inflection for the absorption enhancement, which runs up to the maximum under $w=0.7$ $\mu \mathrm{m}$. On the other hand, we also show in the inset of Figure $2 \mathrm{a}$ the influence of the length $l$ of cut wires to the frequency of electric dipolar mode and corresponding peak value of absorption under a fixed width $w=0.7 \mu \mathrm{m}$. Similar to the cases of changing line width of cut wires, we demonstrate the resonance frequency decreases monotonically by increasing $l$, while the absorption reaches a maximum value of $50 \%$ when $l=$ $5.0 \mu \mathrm{m}$. The discussions to the simple cut-wire structure confirm that the $50 \%$ maximum absorption enhancement still holds for the excitation of electric dipolar mode in a graphene sheet. ${ }^{38}$ In the view of experiments, graphene generally needs to be transferred onto some substrate for patterning. Hence, we studied the scattering responses of a cut-wire array (with the same geometry as that in Figure $2 \mathrm{a}$ ) on top of a $0.5 \mu \mathrm{m}$ thick substrate, the dielectric constant of which was taken as $\epsilon_{\mathrm{r}}=2.4$. As can be seen from Figure 3, the resonant frequency shifts to lower frequency as expected because of the introduction of the substrate, but the maximum absorption keeps $50 \%$.

Actually, the $50 \%$ maximum absorption can be understood by employing the transfer matrix formalism for a conductive 


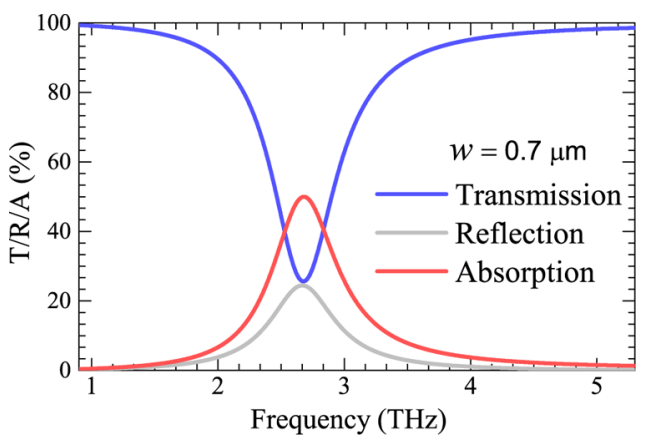

Figure 3. Transmission, reflection, and the absorption of a graphene cut-wire array (theoretical graphene data: $\alpha=58.86 \mathrm{GHz} / \Omega, \gamma=2$ $\mathrm{THz} ; w=0.7 \mu \mathrm{m})$ with substrate.

sheet $^{39}$ as follows: since the graphene cut-wires are of deep subwavelength, by neglecting high-order scatterings, we have the absorption $(A)$ of a free-standing conductive sheet (effective complex conductivity is $\sigma$ ) as follows,

$$
\begin{aligned}
A & =1-|r|^{2}-|t|^{2} \\
& =1-\left|\frac{\sigma \eta_{0}}{2+\sigma \eta_{0}}\right|^{2}-\left|\frac{2}{2+\sigma \eta_{0}}\right|^{2} \\
& =\frac{4}{\left(\left[4+\left(\sigma_{\mathrm{i}} \eta_{0}\right)^{2}\right] / \sigma_{\mathrm{r}} \eta_{0}\right)+\sigma_{\mathrm{r}} \eta_{0}+4} \\
& \leq \frac{1}{2}
\end{aligned}
$$

where $\eta_{0}$ represents the characteristic impedance of vacuum, $r$ and $t$ are the complex scattering coefficients, $\sigma_{\mathrm{r}}$ and $\sigma_{\mathrm{i}}$ are the real and imaginary part of $\sigma$, respectively. We can find the absorption gets the maximum, that is, $50 \%$, when $\sigma_{\mathrm{r}} \eta_{0}=2$ and $\sigma_{\mathrm{i}}=0$.

The widely accepted retrieval method ${ }^{50,51}$ for the extraction of effective EM parameters from complex $T$ and $R$ coefficients, provides a very intuitive route to understand the EM properties of metamaterials. For better understanding to the $\mathrm{THz}$ response of the proposed design, we applied a recently proposed sheet retrieval method, ${ }^{52}$ which is perfectly suitable for our $2 \mathrm{D}$ graphene structure, and got the effective electric surface conductivity $\sigma_{\|}^{\mathrm{e}}$, which can be expressed in the form of complex scattering coefficients $r$ and $t$ as

$$
\sigma_{\|}^{\mathrm{e}}=\frac{2}{\zeta}\left(\frac{1-r-t}{1+r+t}\right)
$$

with $\zeta$ representing the wave impedance. The extracted complex surface conductivity of the graphene meta-surface for the case of $w=0.7 \mu \mathrm{m}$ is shown in Figure 4 (blue lines, light dashed and dark solid curves show the real and imaginary parts, respectively). It obviously shows a Lorentz resonant response around $3.30 \mathrm{THz}$ on the effective electric conductivity spectrum, which confirms the excitation of electric dipolar mode. The extracted magnetic conductivity does not show any resonant feature around this electric resonance, so we have not included the corresponding result here. So far, we investigated the graphene meta-surface with the theoretical data applied, that is, Drude weight $\alpha=58.86 \mathrm{GHz} / \Omega$, collision frequency $\gamma=$ $2 \mathrm{THz}$. Here, we also consider the meta-surface made from some experimentally practical graphene, that is, Yan et al. graphene with $\alpha=76.0 \mathrm{GHz} / \Omega$ and $\gamma=9.8 \mathrm{THz}$. The same

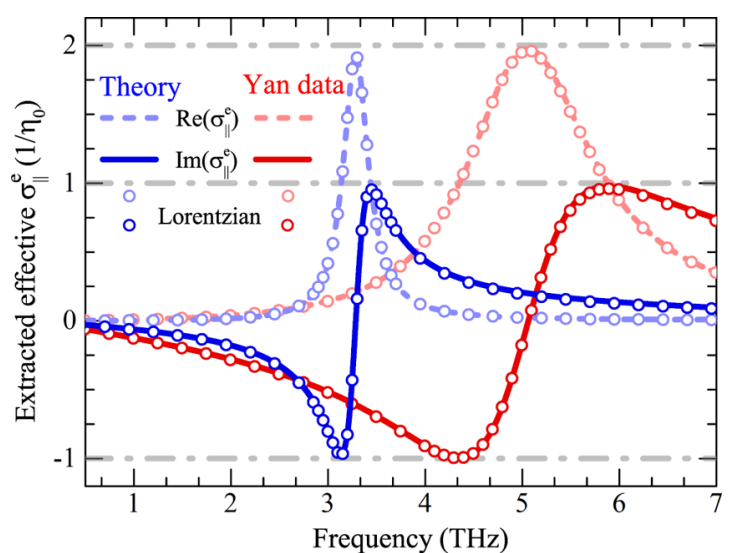

Figure 4. Extracted effective surface conductivities for graphene cutwire arrays with theoretical graphene data $(\alpha=58.86 \mathrm{GHz} / \Omega, \gamma=2$ $\mathrm{THz} ; w=0.7 \mu \mathrm{m})$ and Yan et al. graphene data $(\alpha=76.0 \mathrm{GHz} / \Omega, \gamma=$ $9.8 \mathrm{THz} ; w=2.6 \mu \mathrm{m}$ ), respectively. Lorentzian fittings of the resonant conductivity responses are plotted on the extracted curves.

procedure for studying the influence of line width $w$ was performed and the maximum absorption enhancement $50 \%$ happens at $w=2.6 \mu \mathrm{m}$ (results not shown). The retrieved effective electric conductivity for Yan et al. graphene metasurface with $w=2.6 \mu \mathrm{m}$ is presented in Figure 4 from which the electric resonance is found to shift to $5.06 \mathrm{THz}$ for this case. An interesting demonstration is that the effective conductivity amplitudes (both real and imaginary parts) for the metasurfaces of theoretical graphene and Yan et al. graphene are nearly the same, as indicated by the dashed lines in Figure 4. It is also seen from Figure 4 that the condition $\sigma \eta_{0}=2$ is almost fulfilled for the maximum $50 \%$ absorption at the resonant frequencies in both the theoretical graphene and Yan et al. graphene cases.

Since the effective electric surface conductivity of the graphene meta-surface shows a Lorentz response, we attempted a Lorentzian function to fit the extracted effective conductivity for quantitative descriptions of the electric dipolar resonances (circles on top of the extracted curves in Figure 4):

$$
\sigma_{\|}^{\mathrm{e}}=\frac{i \kappa f}{f^{2}+i \Gamma f-f_{\mathrm{r}}^{2}}-i \beta f
$$

where $f_{\mathrm{r}}$ is the resonant frequency, $\Gamma$ is the damping frequency, $\kappa$ is the coupling constant, and $\beta$ characterizes the background polarization. The fitted Lorentz factors are $f_{\mathrm{r}}=3.30 \mathrm{THz}, \Gamma=$ $315.0 \mathrm{GHz}, \kappa=606.4 \mathrm{GHz}$ for the theoretical graphene metasurface, and $f_{\mathrm{r}}=5.06 \mathrm{THz}, \Gamma=1554.0 \mathrm{GHz}, \kappa=3048.1 \mathrm{GHz}$ for Yan et al. graphene meta-surface. We found two characters from the fitted Lorentz factors: $\Gamma_{2} / \Gamma_{1} \approx \gamma_{2} / \gamma_{1}$ and $\kappa_{1} / \Gamma_{1} \approx \kappa_{2} /$ $\Gamma_{2}$, where the subscripts 1 and 2 denote the cases of theoretical and Yan et al. graphene, respectively. These two relations imply that (i) the damping frequency of the graphene cut-wire is determined by the collision frequency of graphene; and (ii) graphene meta-surfaces with same absorption ( $50 \%$ for both cases) have nearly same resonant amplitude of $\kappa / \Gamma$. The extracted effective surface conductivities (curves) and Lorentzian fitting (circles) for a graphene cut-wire array with substrate are shown in Figure 5, we notice the relation $\sigma \eta_{0}=2$ is also fulfilled for supporting the maximum $50 \%$ absorption at the new resonant frequency $2.68 \mathrm{THz}$.

To further investigate the graphene meta-surface with different surface conductivities, we comparatively studied the 


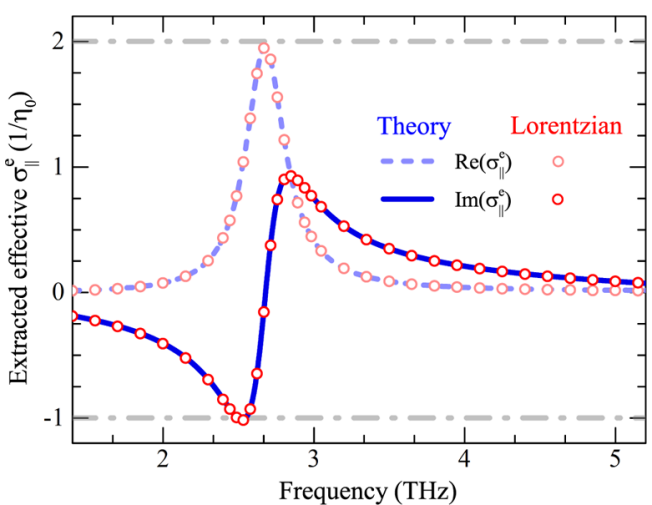

Figure 5. Extracted effective surface conductivities (curves) and Lorentzian fitting (circles) for graphene cut-wire array (theoretical graphene data: $\alpha=58.86 \mathrm{GHz} / \Omega, \gamma=2 \mathrm{THz} ; w=0.7 \mu \mathrm{m})$ with substrate.

graphene meta-surface by changing the values of $\alpha$ and $\gamma$ between that of the two sets of well-known experimental data, that is, $\mathrm{Li}$ et al. graphene $(\alpha=19.9 \mathrm{GHz} / \Omega, \gamma=29.4 \mathrm{THz})$ and Yan et al. graphene. ${ }^{13,23,26}$ The line width of cut-wires $w$ is set as $2.6 \mu \mathrm{m}$ for the discussions in this section. The false-color maps of the extracted electric surface conductivity are plotted in Figure 6a [imaginary part: $\left.\operatorname{Im}\left(\sigma_{\|}^{\mathrm{e}}\right)\right]$ and Figure $6 \mathrm{~b}$ [real part: $\left.\operatorname{Re}\left(\sigma_{\|}^{\mathrm{e}}\right)\right]$, respectively. The experimental parameters of graphene change from the Li et al. data (denoted as (1) to the Yan et al. data (denoted as (3) in two steps: by decreasing collision frequency $\gamma($ (1) $\rightarrow$ (2) ) and increasing Drude weight $\alpha$ (2) $\rightarrow$ (3)),

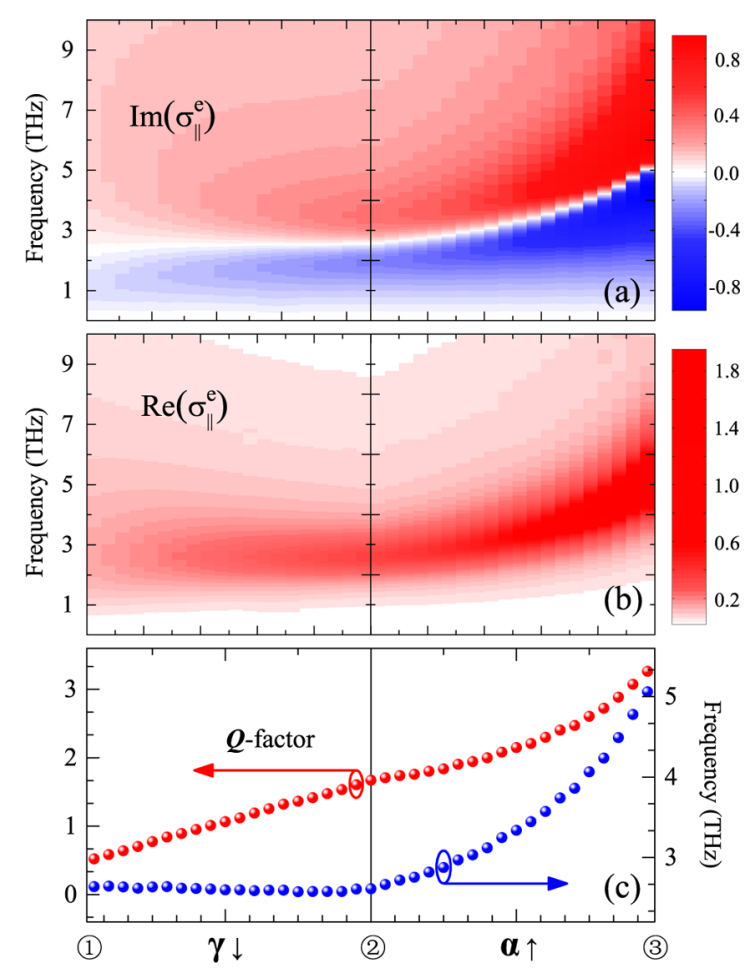

Figure 6. Spectra of imaginary (a) and real (b) part of extracted effective surface conductivities as a function of the graphene Drude weight $\alpha$ and the collision frequency $\gamma$. The parameters of graphene change from the $\mathrm{Li}$ et al. data to the Yan et al. data in two steps: first decrease $\gamma($ (1) $\rightarrow$ (2) ) and then increase $\alpha$ (2) $\rightarrow$ (3)). (c) Q-factors and resonant frequencies of graphene cut-wire arrays with different Drude weights and collision frequencies. where (2) represents a set of hypothetic graphene data with $\alpha=$ $19.9 \mathrm{GHz} / \Omega$ and $\gamma=9.8 \mathrm{THz}$. From (1) $\rightarrow$ (3) we can see the strength of the electric dipolar resonance increases by improving the quality of graphene (from the $\mathrm{Li}$ et al. data in 2008 to Yan et al. data in 2012). This can be further confirmed quantitatively from Figure $6 c$, in which we plotted the resonant frequencies $\left(f_{\mathrm{r}}\right)$ and $Q$-factors $\left(f_{\mathrm{r}} / \Gamma\right)$ of graphene cut-wire arrays: (i) the resonant frequency keeps nearly the same when decreasing the collision frequency, however, the linearly changing of the damping frequency makes the $Q$-factor increases constantly; (ii) the damping frequency keeps nearly the same when increasing the Drude weight, however, the blue shift of the resonant frequency makes the $Q$-factor increase continuously, both of which doubled during the increase of the Drude weight.

Finally, an important concern we need to mention here is that even though we have devoted our attention to the impacts of geometric and material parameters of graphene on $\mathrm{THz}$ response of patterned graphene meta-surface, there may exist many other novel mechanisms contributing to the EM responses of graphene plasmonic structures particularly when the scales are down to nanometer regime. ${ }^{53-55}$ For example, intrinsic optical phonons of graphene, scatterings from the edges as well as surface polar phonons in substrate like $\mathrm{SiO}_{2}$ may lead to a significantly modified plasmon dispersion and damping, edge states may cause a red-shift and broadening of the plasmon resonance in doped graphene nanodisks with zigzag edges, and nonlocal response arised from spatial inhomogeneity will also affect plasmonic resonances in graphene nanostructures. A very recent study also reported the progress in achieving high confinement and low levels of plasmon damping by sandwiching graphene in an exceptionally clean environment with hexagonal boron nitride films. ${ }^{56}$ Overall, it should be crucial to take into account all these factors in the studies of nanoscale graphene structures.

In summary, we exploited graphene cut-wire arrays to realize tunable meta-surfaces responding in the terahertz regime. The enhanced 50\% maximum absorption was studied by optimizing the geometry of graphene cut-wires. The sheet retrieval method was employed to study two graphene meta-surfaces with different graphene's factors but showing the same absorption enhancement, and it is found (i) the collision frequency of graphene determines the damping frequency of the graphene meta-surface; and (ii) the resonant amplitude $(\kappa / \Gamma)$ can be used to describe the absorption in a quantitative way. Systematic investigations on graphene's factors show that the improvements in preparing high-quality graphene have got remarkable progresses in increasing the $Q$-factors of graphene metamaterials. Our results on the terahertz absorption enhancement and tunability of graphene sheet with the excitaion of electric resonance may have potential applications for terahertz and infrared band graphene photonics and optoelectronics.

\section{AUTHOR INFORMATION}

\section{Corresponding Authors}

*E-mail: phyfan@nwpu.edu.cn.

*E-mail: nhshen@ameslab.gov.

\section{Notes}

The authors declare no competing financial interest. 


\section{ACKNOWLEDGMENTS}

Work at Ames Laboratory was partially supported by the U.S. Department of Energy (Basic Energy Sciences, Division of Materials Sciences and Engineering) under Contract No. DEAC02-07CH11358. This work was partially supported by European Research Council under the ERC Advanced Grant No. 320081 (PHOTOMETA). Y.F. acknowledges the China Scholarship Council (No. 201206260055) for financial support.

\section{REFERENCES}

(1) Smith, D. R.; Pendry, J. B.; Wiltshire, M. C. K. Metamaterials and negative refractive index. Science 2004, 305, 788-792.

(2) Soukoulis, C. M.; Wegener, M. Past achievements and future challenges in the development of three-dimensional photonic metamaterials. Nat. Photonics 2011, 5, 523-530.

(3) Kildishev, A. V.; Boltasseva, A.; Shalaev, V. M. Planar photonics with metasurfaces. Science 2013, 339, 1232009.

(4) Yu, N.; Capasso, F. Flat optics with designer metasurfaces. Nat. Mater. 2014, 13, 139-150.

(5) Yu, N.; Genevet, P.; Kats, M. A.; Aieta, F.; Tetienne, J.-P.; Capasso, F.; Gaburro, Z. Light propagation with phase discontinuities: Generalized laws of reflection and refraction. Science 2011, 334, 333337.

(6) Ni, X.; Emani, N. K.; Kildishev, A. V.; Boltasseva, A.; Shalaev, V. M. Broadband light bending with plasmonic nanoantennas. Science 2012, 335, 427.

(7) Sun, S.; He, Q.; Xiao, S.; Xu, Q.; Li, X.; Zhou, L. Gradient-index meta-surfaces as a bridge linking propagating waves and surface waves. Nat. Mater. 2012, 11, 426-431.

(8) Yin, X.; Ye, Z.; Rho, J.; Wang, Y.; Zhang, X. Photonic spin hall effect at metasurfaces. Science 2013, 339, 1405-1407.

(9) Shitrit, N.; Yulevich, I.; Maguid, E.; Ozeri, D.; Veksler, D.; Kleiner, V.; Hasman, E. Spin-optical metamaterial route to spincontrolled photonics. Science 2013, 340, 724-726.

(10) Zheludev, N. I.; Kivshar, Y. S. From metamaterials to metadevices. Nat. Mater. 2012, 11, 917-924.

(11) Kats, M. A.; Sharma, D.; Lin, J.; Genevet, P.; Blanchard, R.; Yang, Z.; Qazilbash, M. M.; Basov, D. N.; Ramanathan, S.; Capasso, F. Ultra-thin perfect absorber employing a tunable phase change material. Appl. Phys. Lett. 2012, 101, 221101.

(12) Bonaccorso, F.; Sun, Z.; Hasan, T.; Ferrari, A. C. Graphene photonics and optoelectronics. Nat. Photonics 2010, 4, 611-622.

(13) Yan, H.; Li, X.; Chandra, B.; Tulevski, G.; Wu, Y.; Freitag, M.; Zhu, W.; Avouris, P.; Xia, F. Tunable infrared plasmonic devices using graphene/insulator stacks. Nat. Nanotechnol. 2012, 7, 330-334.

(14) García de Abajo, F. J. Graphene plasmonics: challenges and opportunities. ACS Photonics 2014, 1, 135-152.

(15) Low, T.; Avouris, P. Graphene plasmonics for terahertz to midinfrared applications. ACS Nano 2014, 8, 1086-1101.

(16) Mikhailov, S. A.; Ziegler, K. New electromagnetic mode in graphene. Phys. Rev. Lett. 2007, 99, 016803.

(17) Jablan, M.; Buljan, H.; Soljačić, M. Plasmonics in graphene at infrared frequencies. Phys. Rev. B 2009, 80, 245435.

(18) Chen, J.; Badioli, M.; Alonso-González, P.; Thongrattanasiri, S.; Huth, F.; Osmond, J.; Spasenović, M.; Centeno, A.; Pesquera, A.; Godignon, P.; Zurutuza Elorza, A.; Camara, N.; García de Abajo, F. J.; Hillenbrand, R; Koppens, F. H. L. Optical nano-imaging of gatetunable graphene plasmons. Nature 2012, 487, 77-81.

(19) Fei, Z.; Rodin, A. S.; Andreev, G. O.; Bao, W.; McLeod, A. S.; Wagner, M.; Zhang, L. M.; Zhao, Z.; Thiemens, M.; Dominguez, G.; Fogler, M. M.; Neto, A. H. C.; Lau, C. N.; Keilmann, F.; Basov, D. N. Gate-tuning of graphene plasmons revealed by infrared nano-imaging. Nature 2012, 487, 82-85.

(20) Gerber, J. A.; Berweger, S.; OąŕCallahan, B. T.; Raschke, M. B. Phase-resolved surface plasmon interferometry of graphene. Phys. Rev. Lett. 2014, 113, 055502.

(21) Vakil, A.; Engheta, N. Transformation optics using graphene. Science 2011, 332, 1291-1294.
(22) Koppens, F. H. L.; Chang, D. E.; García de Abajo, F. J. Graphene Plasmonics: A platform for strong lightĺCmatter interactions. Nano Lett. 2011, 11, 3370-3377.

(23) Li, Z. Q.; Henriksen, E. A.; Jiang, Z.; Hao, Z.; Martin, M. C.; Kim, P.; Stormer, H. L.; Basov, D. N. Dirac charge dynamics in graphene by infrared spectroscopy. Nat. Phys. 2008, 4, 532-535.

(24) Wang, F.; Zhang, Y.; Tian, C.; Girit, C.; Zettl, A.; Crommie, M.; Shen, Y. R. Gate-variable optical transitions in graphene. Science 2008, 320, 206-209.

(25) Horng, J.; Chen, C.-F.; Geng, B.; Girit, C.; Zhang, Y.; Hao, Z.; Bechtel, H. A.; Martin, M.; Zettl, A.; Crommie, M. F.; Shen, Y. R.; Wang, F. Drude conductivity of Dirac fermions in graphene. Phys. Rev. B 2011, 83, 165113.

(26) Tassin, P.; Koschny, T.; Soukoulis, C. M. Graphene for terahertz applications. Science 2013, 341, 620-621.

(27) Nair, R. R.; Blake, P.; Grigorenko, A. N.; Novoselov, K. S.; Booth, T. J.; Stauber, T.; Peres, N. M. R.; Geim, A. K. Fine structure constant defines visual transparency of graphene. Science 2008, 320, 1308.

(28) Hess, O.; Pendry, J. B.; Maier, S. A.; Oulton, R. F.; Hamm, J. M.; Tsakmakidis, K. L. Active nanoplasmonic metamaterials. Nat. Mater. 2012, 11, 573-584.

(29) Kauranen, M.; Zayats, A. V. Nonlinear plasmonics. Nat. Photonics 2012, 6, 737-748.

(30) Thongrattanasiri, S.; Koppens, F. H. L.; García de Abajo, F. J. Complete optical absorption in periodically patterned graphene. Phys. Rev. Lett. 2012, 108, 047401.

(31) Alaee, R.; Farhat, M.; Rockstuhl, C.; Lederer, F. A perfect absorber made of a graphene micro-ribbon metamaterial. Opt. Express 2012, 20, 28017-28024.

(32) He, S.; Zhang, X.; He, Y. Graphene nano-ribbon waveguides of record-small mode area and ultra-high effective refractive indices for future VLSI. Opt. Express 2013, 21, 30664-30673.

(33) Chen, P.-Y.; Alù, A. Atomically thin surface cloak using graphene monolayers. ACS Nano 2011, 5, 5855-5863.

(34) Cheng, H.; Chen, S.; Yu, P.; Li, J.; Deng, L.; Tian, J. Midinfrared tunable optical polarization converter composed of asymmetric graphene nanocrosses. Opt. Lett. 2013, 38, 1567-1569.

(35) Liu, P.; Cai, W.; Wang, L.; Zhang, X.; Xu, J. Tunable terahertz optical antennas based on graphene ring structures. Appl. Phys. Lett. 2012, 100, 153111.

(36) Wang, W. Plasmons and optical excitations in graphene rings. J. Phys.: Condens. Matter 2012, 24, 402202.

(37) Papasimakis, N.; Thongrattanasiri, S.; Zheludev, N. I.; García de Abajo, F. J. The magnetic response of graphene split-ring metamaterials. Light: Sci. Appl. 2013, 2, e78.

(38) Fan, Y.; Wei, Z.; Zhang, Z.; Li, H. Enhancing infrared extinction and absorption in a monolayer graphene sheet by harvesting the electric dipolar mode of split ring resonators. Opt. Lett. 2013, 38, $5410-5413$.

(39) Fan, Y.; Zhang, F.; Zhao, Q.; Wei, Z.; Li, H. Tunable terahertz coherent perfect absorption in a monolayer graphene. Opt. Lett. 2014, 39, 6269-6272.

(40) Fan, Y.; Wei, Z.; Li, H.; Chen, H.; Soukoulis, C. M. Photonic band gap of a graphene-embedded quarter-wave stack. Phys. Rev. B 2013, 88, 241403(R).

(41) Zhang, S.; Genov, D.; Wang, Y.; Liu, M.; Zhang, X. Plasmoninduced transparency in metamaterials. Phys. Rev. Lett. 2008, 101, 047401.

(42) Liu, N.; Langguth, L.; Weiss, T.; Kästel, J.; Fleischhauer, M.; Pfau, T.; Giessen, H. Plasmonic analogue of electromagnetically induced transparency at the Drude damping limit. Nat. Mater. 2009, 8, $758-762$.

(43) Tassin, P.; Zhang, L.; Zhao, R.; Jain, A.; Koschny, T.; Soukoulis, C. M. Electromagnetically induced transparency and absorption in metamaterials: the radiating two-oscillator model and its experimental confirmation. Phys. Rev. Lett. 2012, 109, 187401.

(44) Grady, N. K.; Heyes, J. E.; Chowdhury, D. R.; Zeng, Y.; Reiten, M. T.; Azad, A. K.; Taylor, A. J.; Dalvit, D. A. R.; Chen, H.-T. 
Terahertz metamaterials for linear polarization conversion and anomalous refraction. Science 2013, 340, 1304-1307.

(45) Novotny, L. From near-field optics to optical antennas. Phys. Today 2011, 64, 47-52.

(46) Wunsch, B.; Stauber, T.; Sols, F.; Guinea, F. Dynamical polarization of graphene at finite doping. New J. Phys. 2006, 8, 318.

(47) Hwang, E. H.; Das Sarma, S. Dielectric function, screening, and plasmons in two-dimensional graphene. Phys. Rev. B 2007, 75, 205418.

(48) Gusynin, V. P.; Sharapov, S. G.; Carbotte, J. P. Magneto-optical conductivity in graphene. J. Phys.: Condens. Matter 2007, 19, 026222.

(49) Shen, N.-H.; Tassin, P.; Koschny, T.; Soukoulis, C. M. Comparison of gold- and graphene-based resonant nanostructures for terahertz metamaterials and an ultrathin graphene-based modulator. Phys. Rev. B 2014, 90, 115437.

(50) Smith, D. R.; Schultz, S.; Markoš, P.; Soukoulis, C. M. Determination of effective permittivity and permeability of metamaterials from reflection and transmission coefficients. Phys. Rev. B 2002, 65, 195104.

(51) Chen, X.; Grzegorczyk, T. M.; Wu, B.-I.; Pacheco, J.; Kong, J. A. Robust method to retrieve the constitutive effective parameters of metamaterials. Phys. Rev. E 2004, 70, 016608.

(52) Tassin, P.; Koschny, T.; Soukoulis, C. M. Effective material parameter retrieval for thin sheets: Theory and application to graphene, thin silver films, and single-layer metamaterials. Physica $B$ 2012, 407, 4062-4065.

(53) Yan, H.; Low, T.; Zhu, W.; Wu, Y.; Freitag, M.; Li, X.; Guinea, F.; Avouris, P.; Xia, F. Damping pathways of mid-infrared plasmons in graphene nanostructures. Nat. Photonics 2013, 7, 394-399.

(54) Christensen, T.; Wang, W.; Jauho, A.-P.; Wubs, M.; Asger Mortensen, N. Classical and quantum plasmonics in graphene nanodisks: the role of edge states. arXiv:1407.3920, 2014.

(55) Fallahi, A.; Low, T.; Tamagnone, M.; Perruisseau-Carrier J. Nonlocal electromagnetic response of graphene nanostructures. arXiv:1410.1725 2014.

(56) Woessner, A.; Lundeberg, M. B.; Gao, Y.; Principi, A.; AlonsoGonzález, P.; Carrega, M.; Watanabe, K.; Taniguchi, T.; Vignale, G.; Polini, M.; Hone, J.; Hillenbrand, R.; Koppens, F. H. L. Highly confined low-loss plasmons in graphene-boron nitride heterostructures. arXiv:1409.5674 2014. 